\title{
Nuclear parton distributions at next to leading order.
}

\author{
D. de Florian, R. Sassot状 \\ Departamento de Física, Facultad de Ciencias Exactas y Naturales, \\ Universidad de Buenos Aires, Pabellón I, Ciudad Universitaria (1428) Buenos Aires, Argentina
}

(Dated: November 16, 2018)

\begin{abstract}
We perform a next to leading order QCD global analysis of nuclear deep inelastic scattering and Drell-Yan data using the convolution approach to parameterize nuclear parton densities. We find both a significant improvement in the agreement with data compared to previous extractions, and substantial differences in the scale dependence of nuclear effects compared to leading order analyses.
\end{abstract}

PACS numbers: $13.60 \mathrm{Hb}, 12.38 . \mathrm{Bx}, 24.85 .+\mathrm{p}$

\section{INTRODUCTION}

Ever since the discovery that quark and gluons in bound nucleons show momentum distributions noticeably different to those measured in free or less bound nucleons [1], two decades ago, the precise determination of nuclear parton densities has awakened growing attention, driving both increasingly precise and comprehensive nuclear structure functions measurements [2], and more refined theoretical understanding of the underlying physics.

The precise knowledge of nuclear parton distribution functions (nPDF) is not only required for a deeper understanding of the mechanisms associated to nuclear binding from a QCD improved parton model perspective, but it is also the starting point for the analyses of a wide variety of future and ongoing high energy physics experiments, such as heavy ion collisions at RHIC [3] , proton-nucleus collisions to be performed at LHC [4], or neutrino-nucleus interactions in long baseline neutrino experiments [5], for example. Consequently, the accuracy of nPDF is rapidly evolving into a key issue in many areas of particle physics.

From the point of view of perturbative QCD, the extraction of $\mathrm{nPDF}$ can be done in close analogy to what is done for free nucleons: they are considered as non perturbative inputs, to be inferred from data, whose relation to the measured observables and energy scale dependence are computed order by order in perturbation theory. Although one can not discard larger higher twist power corrections than in the case of free nucleons, or even some sort of nuclear recombination effect, factorization and universality of $\mathrm{nPDF}$ are expected to hold in a very good approximation, over a wide kinematical range in the present experiments.

At variance with nucleon PDF, which driven by the demand of more and more precise predictions, have at-

*Partially supported by Conicet, ANPCyT, UBACyT and Fundación Antorchas

†Electronic address: deflo@df.uba.ar, sassot@df.uba.ar tained in the last years an impressive degree of accuracy and refinement, nPDF extractions are in a considerably earlier stage of the development. Not only the number and diversity of nuclear data is much more reduced, but the analyses are restricted to leading order accuracy (LO), with rather crude parameterizations of nuclear effects which lead to poor $\chi^{2} /$ d.o.f values in global QCD fits to data [6, 7].

There are also some caveats inherent to the particular approaches implemented so far, that define nPDF in terms of nucleon PDF and a multiplicative nuclear correction factor at a given initial energy scale, from where they are evolved. In the first place, nPDF defined in this way have their momentum fraction per nucleon restricted to be less or equal than unity, what excludes from the description, and also from the evolution, a portion of its natural kinematical range. Of course, unless one is specifically interested in that region, this approximation is expected to imply a minor correction, although one can not discard the excluded region as a source of new and interesting information.

Much more problematic in these approaches is that the actual shape of the nuclear correction factor required to reproduce accurately the data implies very capricious functions, with a large number of parameters, and which in practice precludes the numerical computation of the scale dependence at next to leading order accuracy (NLO). The actual computation of structure functions and evolution equations at this order implies several convolution integrals very difficult to evaluate unless Mellin transform techniques are applied.

In this paper we show that a much more convenient alternative to deal with nuclear effects is to define nPDF using a convolution approach. In such framework the free nucleon parton densities are convoluted with very simple weight functions that parameterize nuclear effects. The convolution method naturally takes into account the actual range of nucleon momentum fractions, allows via Mellin transform techniques a straightforward numerical evaluation of the NLO scale dependence, lead to extraordinarily accurate $\mathrm{nPDF}$ with relatively few parameters, and finally, allow to interpret the nuclear modifications 
in terms of a very simple mechanism of rebalance of momentum fractions between the distributions. The success of the convolution approach comes from the fact that the momentum fraction dependence of nuclear effects is strongly correlated to that of partons in free nucleons, as shown in re-scaling models [8], feature which is explicitly included by the convolution.

As an example of feasibility of the convolution approach we obtain for the first time a full NLO extraction of $\mathrm{nPDF}$ from a large number of nuclear DIS [13, 14, 15, 16] and Drell Yan data 17. We also asses the differences between the LO and NLO extractions, finding that although the quality of fits to present data is comparable in both approximations, there are important differences between the $Q^{2}$ dependence of the nuclear correction factors using either $\mathrm{LO}$ or NLO extractions. This, for example, questions the use of LO factors with NLO nucleon parton densities to generate nPDF in NLO computations, as it is common practice.

In the following section we summarize the main motivation and features of the convolution approach. In the third section we present the details and outcome of the NLO and LO nPDF extractions, comparing our results with previous LO analyses. In the last section we discuss the differences between LO and NLO extractions of $\mathrm{nPDF}$, computing the one hadron production cross section with them as and example, and present our conclusions.

\section{NPDF}

The description of DIS processes off nuclear targets, $e A \rightarrow e^{\prime} X$, is customarily done in terms of the hard scale $Q^{2}$, defined as minus the virtuality of the exchanged photon, and a scaling variable $x_{A}$, analogue to the Bjorken variable used in DIS off nucleons,

$$
Q^{2} \equiv-q^{2}, \quad x_{A} \equiv \frac{Q^{2}}{2 p_{A} \cdot q},
$$

respectively. Here $p_{A}$ is the target nucleus momentum and consequently, $x_{A}$ is kinematically restricted to $0<x_{A}<1$, just as the standard Bjorken variable. Alternatively, one can define another scaling variable $x_{N} \equiv A x_{A}$, where $A$ is the mass number of the nucleus. Under the assumption that the nucleus momentum $p_{A}$ is evenly distributed between that of the constituent nucleons $p_{N}=p_{A} / A$, this variable resembles the Bjorken variable corresponding to the scattering off free nucleons, $x_{N} \equiv Q^{2} /\left(2 p_{N} \cdot q\right)$. However in nuclear scattering context of course it spans the interval $0<x_{N}<A$, by definition, and reflecting the fact that a parton may in principle carry more than the average nucleon momentum.

When discussing nPDF the usual approach is to propose a very simple relation between the parton distribution of a proton bound in the nucleus, $f_{i}^{A}$, and those for free protons $f_{i}$,

$$
f_{i}^{A}\left(x_{N}, Q_{0}^{2}\right)=R_{i}\left(x_{N}, Q_{0}^{2}, A, Z\right) f_{i}\left(x_{N}, Q_{0}^{2}\right),
$$

in terms of a multiplicative nuclear correction factor $R_{i}\left(x_{N}, Q^{2}, A, Z\right)$, specific for a given nucleus $(A, Z)$, parton flavor $i$, and initial energy scale $Q_{0}^{2}$. This description is convenient since the ratio $R_{i}\left(x_{N}, Q^{2}, A, Z\right)$ compares directly the parton densities with and without nuclear effects, and is closely related to the most usual nuclear DIS observables, which are the ratios between the nuclear and deuterium structure functions. Indeed, we will use this picture to simplify the presentation of the output of our analysis. However, this is not the best suited way to parameterize the effects in the intermediate steps of the analysis, nor the best alternative for higher order numerical computations, as it was mentioned previously.

Since $f_{i}\left(x_{N}, Q^{2}\right)$ are defined for $0<x_{N}<1$, nPDF defined as in Eq.(2) are a priori restricted to that same range at the initial scale. In most analyses the evolution equations are also constrained to $0<x_{N}<1$, loosing the possibility of analyzing nuclear effects beyond $x_{N}>1$ at any other scale. Notice that even if nPDF were constrained to be zero for $x_{N}>1$ at a given scale, the complete evolution equations would produce non-zero values for other scales in the range of $x_{N}$ neglected by the approach, leading to a possible violation of momentum conservation. From another side, the direct parameterization of the ratios $R_{i}\left(x_{N}, Q^{2}, A, Z\right)$ require many parameters and even $x_{N}$-dependencies whose Mellin moments can not be put into a closed expression.

A much more adequate alternative to Eq.(2) is to relate nPDF to standard PDF by means of a convolution

$$
f_{i}^{A}\left(x_{N}, Q_{0}^{2}\right)=\int_{x_{N}}^{A} \frac{d y}{y} W_{i}(y, A, Z) f_{i}\left(\frac{x_{N}}{y}, Q_{0}^{2}\right) .
$$

where the weight function $W_{i}(y, A, Z)$ parameterize now the nuclear effects and can be thought as the effective density of nucleons within the nucleus carrying a fraction $y / A$ of its longitudinal momentum. Besides allowing the full kinematical range for $\mathrm{nPDF}$, this kind of approach has been shown to lead to remarkably good parameterizations of nuclear effects with very few parameters, and with a smooth $A$-dependence [9]. For example, neglecting nuclear effects, the effective nucleon density is just $W_{i}(y, A, Z)=A \delta(1-y)$, and simple shifts in the momentum fraction carried by nucleons $W_{i}(y, A, Z)=A \delta(1-y-\epsilon)$ have been shown to reproduce in a very good approximation many features of the known nuclear modifications to structure functions.

The convolution approach also allows to perform the evolution in Mellin space, which is much more convenient numerically, and almost mandatory for NLO accuracy. Defining $y_{A} \equiv \frac{y}{A}$ Eq.(3) reads

$$
f_{i}^{A}\left(A x_{A}, Q_{0}^{2}\right)=\int_{x_{A}}^{1} \frac{d y_{A}}{y_{A}} W_{i}\left(A y_{A}, A, Z\right) f_{i}\left(\frac{x_{A}}{y_{A}}, Q_{0}^{2}\right)
$$

and in Mellin space, i.e. taking moments in both sides of Eq.(4),

$$
\hat{f}_{i}^{A}\left(N, Q_{0}^{2}\right)=\hat{W}_{i}(N, A, Z) \hat{f}_{i}\left(N, Q_{0}^{2}\right)
$$


where

$$
\hat{f}_{i}^{A}\left(N, Q_{0}^{2}\right) \equiv \int_{0}^{1} d x x^{N-1} f_{i}^{A}\left(A x, Q_{0}^{2}\right)
$$

and similarly for $\hat{f}_{i}\left(N, Q_{0}^{2}\right)$ with $A=1$, and

$$
\hat{W}_{i}(N, A, Z) \equiv \int_{0}^{1} d x x^{N-1} W_{i}(A x, A, Z) .
$$

Notice that the moments are defined in terms of the correct scaling variable $x_{A}$, and that the evolution of the nPDF moments $\hat{f}_{i}^{A}\left(N, Q_{0}^{2}\right)$ can be managed with a standard evolution code in Mellin space.

Nuclear structure functions are defined as the average of the proper combination of the bound proton and neutron structure functions as

$$
A F_{2}^{A}\left(x, Q^{2}\right)=Z F_{2}^{p / A}\left(x, Q^{2}\right)+(A-Z) F_{2}^{n / A}\left(x, Q^{2}\right),
$$

where both bound nucleon structure functions can be written in terms of the corresponding nuclear parton distributions in Mellin space as

$$
\begin{aligned}
F_{2}^{p / A}\left(N-1, Q^{2}\right)=\sum_{q, \bar{q}} e_{q}^{2}\left\{\hat{f}_{i}^{A}\left(N, Q^{2}\right)\right. \\
\left.+\frac{\alpha_{s}}{2 \pi}\left[C_{q}^{(1)}(N) \hat{f}_{i}^{A}\left(N, Q^{2}\right)+C_{g}^{(1)}(N) \hat{f}_{g}^{A}\left(N, Q^{2}\right)\right]\right\}
\end{aligned}
$$

The first term in the right hand side of Eq. (9) corresponds to the leading-order contribution while the second represents the next-to-leading order correction. Expressions for the NLO quark and gluon coefficients $C_{q}^{(1)}(N)$ and $C_{g}^{(1)}(N)$ can be found in [10]. In our analysis we consider only 3 active flavors and neglect the contribution from heavy quarks to the structure function.

Considering that DIS data does not allow to determine all combinations of flavors, and that nuclear effects may be expected to be isospin invariant in a first approximation, it seems to be reasonable to introduce only three independent $W_{i}(y, A, Z)$ : one for the valence distributions, another for the light sea, and the last for the gluons. In this way

$$
\begin{aligned}
& u_{v}^{A}\left(N, Q_{0}^{2}\right)=\hat{W}_{v}(N, A, Z) u_{v}\left(N, Q_{0}^{2}\right) \\
& d_{v}^{A}\left(N, Q_{0}^{2}\right)=\hat{W}_{v}(N, A, Z) d_{v}\left(N, Q_{0}^{2}\right) \\
& \bar{u}^{A}\left(N, Q_{0}^{2}\right)=\hat{W}_{s}(N, A, Z) \bar{u}\left(N, Q_{0}^{2}\right) \\
& \bar{d}^{A}\left(N, Q_{0}^{2}\right)=\hat{W}_{s}(N, A, Z) \bar{d}\left(N, Q_{0}^{2}\right) \\
& g^{A}\left(N, Q_{0}^{2}\right)=\hat{W}_{g}(N, A, Z) g\left(N, Q_{0}^{2}\right)
\end{aligned}
$$

Notice that in many proton parton densities, and in particular in GRV98 [11], the one will be used in the following, the strange and heavy quark densities vanish at the low initial scale and there is no need to introduce additional weight functions for the latter. The parton distributions for the bound neutron can be obtained from the ones above by isospin symmetry.
An interesting feature in this approach is that since non-singlet combinations of parton densities, as those for valence quarks, evolve independently, and both $\hat{f}_{i}^{A}\left(N, Q^{2}\right)$ and $\hat{f}_{i}\left(N, Q^{2}\right)$ obey the same evolution equations, the moments of the weight function $\hat{W}_{i}(N, A, Z)$ are scale independent 21]. This is not the case for sea or gluon weight functions, since they are affected by singlet evolution resulting in a scale dependence due to quark and gluon mixing and therefore needed to be defined at a particular scale.

Charge, baryon number and momentum conservation imply constraints to be satisfied by the weight functions:

$$
\begin{aligned}
\hat{W}_{v}(N=1, A, Z) & =1 \\
u_{v}^{A}\left(2, Q_{0}^{2}\right)+d_{v}^{A}\left(2, Q_{0}^{2}\right) & \\
+2 \bar{u}^{A}\left(2, Q_{0}^{2}\right)+2 \bar{d}^{A}\left(2, Q_{0}^{2}\right)+g^{A}\left(2, Q_{0}^{2}\right) & =1
\end{aligned}
$$

The best results are obtained using for the valence distributions weight functions like

$$
\begin{aligned}
& W_{v}(y, A, Z)= \\
& \quad A\left[a_{v} \delta\left(1-\epsilon_{v}-y\right)+\left(1-a_{v}\right) \delta\left(1-\epsilon_{v^{\prime}}-y\right)\right] \\
& \quad+n_{v}\left(\frac{y}{A}\right)^{\alpha_{v}}\left(1-\frac{y}{A}\right)^{\beta_{v}}+n_{s}\left(\frac{y}{A}\right)^{\alpha_{s}}\left(1-\frac{y}{A}\right)^{\beta_{s}}
\end{aligned}
$$

where the first two terms may be interpreted as reduction in the parent nucleon longitudinal momentum fraction, and in spite of their simplicity reproduce accurately EMC and Fermi motion effects. Indeed, with just these three parameters the fit reproduces fairly well the large $x_{N}$ data, however it fails to account for antishadowing effects at intermediate $x_{N}$, where valence distributions still dominate.

In order to include antishadowing effects we add the third term in Eq. (13), which induces a small enhancement of the distributions with $n_{v}>0$ and a mild $x_{N}$ dependence given by the parameters $\alpha_{v}$ and $\beta_{v}$. Notice that the convolution integral dilutes the $x_{N}$ dependence producing an effect similar to those predicted by parton recombination models [12]. It would be pointless to try to extract from the data the nuclear modification to valence distributions in the low $x_{N}$ region, since this region is clearly dominated by sea and gluon densities, however the first three terms in Eq. (13) violate charge conservation. In order to remedy this situation we include the forth term, similar to the third but where charge conservation forces $n_{s}<0$. In this case the weight function can not be interpreted as a probability density but as the result of the mechanism that compensate re-scaling and recombination effects. The parameters $\alpha_{s}$ and $\beta_{s}$ are taken to be the parameters used for the sea quark densities, fixed by the low $x_{N}$ behavior of the data, while both $n_{v}$ and $n_{s}$ are fixed by momentum and charge conservation, Eqs.(11) and (12).

For sea quarks and gluons, the argument is just the opposite: the fit to data is not sensitive to any nuclear modification at high or intermediate $x_{N}$, so the best choice 
for sea weight functions is found to be

$$
W_{s}(y, A, Z)=A \delta(1-y)+\frac{a_{s}}{N_{s}}\left(\frac{y}{A}\right)^{\alpha_{s}}\left(1-\frac{y}{A}\right)^{\beta_{s}}
$$

where the first term gives the gluon distribution in free protons and the eulerian function (specifically the parameter $\alpha_{s}$ ) drives the low $x_{N}$ shadowing $\left(a_{s}\right.$ is found to be negative). The three parameters, $a_{s}, \alpha_{s}$ and $\beta_{s}$ are strongly constrained by the structure functions ratios at small $x_{N}$ and the Drell-Yan ratios. $N_{s}$ is just the normalization of the eulerian function, $N_{s}=B\left(\alpha_{s}+2, \beta_{s}+1\right)$. Similarly, for gluons

$$
W_{g}(y, A, Z)=A \delta(1-y)+\frac{a_{g}}{N_{g}}\left(\frac{y}{A}\right)^{\alpha_{g}}\left(1-\frac{y}{A}\right)^{\beta_{g}}
$$

Here, $a_{g}$ is strongly constrained by the $Q^{2}$-dependent $F_{2}^{S n} / F_{2}^{C}$ data, whereas the exponents are taken to be the same as for the sea distributions and $N_{g}=N_{s}$. No significant improvement is found assigning independent parameters for them. Since $n_{v}$ and $n_{s}$ are constrained by momentum conservation in Eqs.(11) and (12), there are 9 independent parameters $\epsilon_{v}, \epsilon_{v^{\prime}}, a_{v}, \alpha_{v}, \beta_{v} a_{s}, \alpha_{s}, \beta_{s}$, and $a_{g}$ for each nucleus. Since no data is available on different isotopes of the same nucleus, in the following we drop the dependence on $Z$. The $A$ dependence of all the parameters can be written

$$
\epsilon_{i}=\gamma_{i}+\lambda_{i} A^{\delta_{i}}
$$

The full parameterization of both the $A$ and $x_{N}$ dependence of nuclear effects imply in principle quite a lot of parameters (27), however the mild $A$ dependence found in some of them allow to reduce their number. For example, $\alpha_{s}, \alpha_{v}$ and $a_{v}$ can be taken as constant in $A$ loosing just a few units in $\chi^{2}$ but eliminating 6 parameters.

\section{RESULTS}

In the following section we present results from the LO and NLO fits to nuclear data using the convolution approach. The data analysed include the most recent NMC and SLAC-E139 DIS structure functions ratios to deuterium and carbon, selecting those measurements corresponding to $Q>1 \mathrm{GeV}$, and also E772 Drell-Yan dilepton cross sections from proton nucleus collisions, as listed in Table 1, rendering a total number of 420 data points.

The parameters were fixed minimizing the function $\chi^{2}$ defined in the customarily way as

$$
\chi^{2} \equiv \sum_{i} \frac{\left(\sigma_{i}^{e x}-\sigma_{i}^{t h}\right)^{2}}{\Delta_{i}^{2}}
$$

where $\sigma_{i}^{e x}$ stands the measured observable, $\sigma_{i}^{t h}$ the corresponding $\mathrm{NLO}(\mathrm{LO})$ estimate, $\Delta_{i}$ the statistical and systematic errors added in quadrature, and the sum runs over all the data points $i$ included in the fit. No artificial weight has been given to any particular subset of data.
TABLE I: Nuclear data included in the fit.

\begin{tabular}{llcc}
\hline \hline Measurement & Collaboration & Refs. & \# data \\
\hline$F_{2}^{H e} / F_{2}^{D}$ & NMC & {$[13]$} & 18 \\
& SLAC-E139 & {$[14]$} & 18 \\
$F_{2}^{B e} / F_{2}^{D}$ & SLAC-E139 & {$[14]$} & 17 \\
$F_{2}^{C} / F_{2}^{D}$ & NMC & {$[13]$} & 18 \\
& SLAC-E139 & {$[14]$} & 7 \\
$F_{2}^{A l} / F_{2}^{D}$ & SLAC-E139 & {$[14]$} & 17 \\
$F_{2}^{C a} / F_{2}^{D}$ & NMC & {$[13]$} & 18 \\
& SLAC-E139 & {$[14]$} & 7 \\
$F_{2}^{F e} / F_{2}^{D}$ & SLAC-E139 & {$[14]$} & 23 \\
$F_{2}^{A g} / F_{2}^{D}$ & SLAC-E139 & {$[14]$} & 7 \\
$F_{2}^{A u} / F_{2}^{D}$ & SLAC-E139 & {$[14]$} & 18 \\
$F_{2}^{B e} / F_{2}^{C}$ & NMC & {$[15]$} & 15 \\
$F_{2}^{A l} / F_{2}^{C}$ & NMC & {$[15]$} & 15 \\
$F_{2}^{C a} / F_{2}^{C}$ & NMC & {$[15]$} & 15 \\
$F_{2}^{F e} / F_{2}^{C}$ & NMC & {$[15]$} & 15 \\
$F_{2}^{P b} / F_{2}^{C}$ & NMC & {$[15]$} & 15 \\
$F_{2}^{S n} / F_{2}^{C}$ & NMC & {$[16]$} & 145 \\
$\sigma_{D Y}^{C} / \sigma_{D Y}^{D}$ & E772 & {$[17]$} & 9 \\
$\sigma_{D Y}^{C a} / \sigma_{D Y}^{D}$ & E772 & {$[17]$} & 9 \\
$\sigma_{D Y}^{F e} / \sigma_{D Y}^{D}$ & E772 & {$[17]$} & 9 \\
$\sigma_{D Y}^{W} / \sigma_{D Y}^{D}$ & E772 & {$[17]$} & 9 \\
\hline Total & & & 420 \\
\hline \hline
\end{tabular}

In order to compute the structure function and DrellYan cross-section for Deuterium, for which we neglect the nuclear corrections, and as a basis to construct the observables for the different nucleus, we use the parton distributions in the free proton as provided by the GRV98 analysis 11. Consequently, we fix the initial scale to $Q_{0}^{2}=0.4 \mathrm{GeV}^{2}\left(0.26 \mathrm{GeV}^{2}\right)$ and the QCD scale (for five flavors) $\lambda_{Q C D}^{(5)}=167 \mathrm{MeV}(132 \mathrm{MeV})$ to $\mathrm{NLO}(\mathrm{LO})$ accuracy. The total $\chi^{2}$ obtained is 300.15 units for the NLO fit and 316.35 for LO one.

The comparison between the data on the ratios of different nuclear structure functions to deuterium and those computed with NLO nPDF is shown in Figure 1 (the LO prediction one is almost indistinguishable). The solid line corresponds to the result of the fit computed at the $Q^{2}$ value of each experimental point, whereas the extrapolation to small $x_{N}$ has been performed using the corresponding $Q^{2}$ of the smallest- $x_{N}$ point. For heavy nuclei, the low $x_{N}$ is mainly constrained by DIS ratios to carbon, as shown in Figure 2. The structure function ratios are useful to determine mainly the valence quark distributions, while Drell Yan data, shown in Figure 3 becomes crucial in order to fix sea quark distributions. The gluon distribution enters the structure function at NLO or through the scale dependence of the parton densities, making very difficult to obtain information about it in DIS experiments. The $Q^{2}$ dependence of $F_{2}^{S n} / F_{2}^{C}$, shown in Figure 5, is the most sensitive available observable to the gluon distribution. Nevertheless, it is worth pointing out that there is still a large uncertainty on this density and data from observables where the gluon distribution 


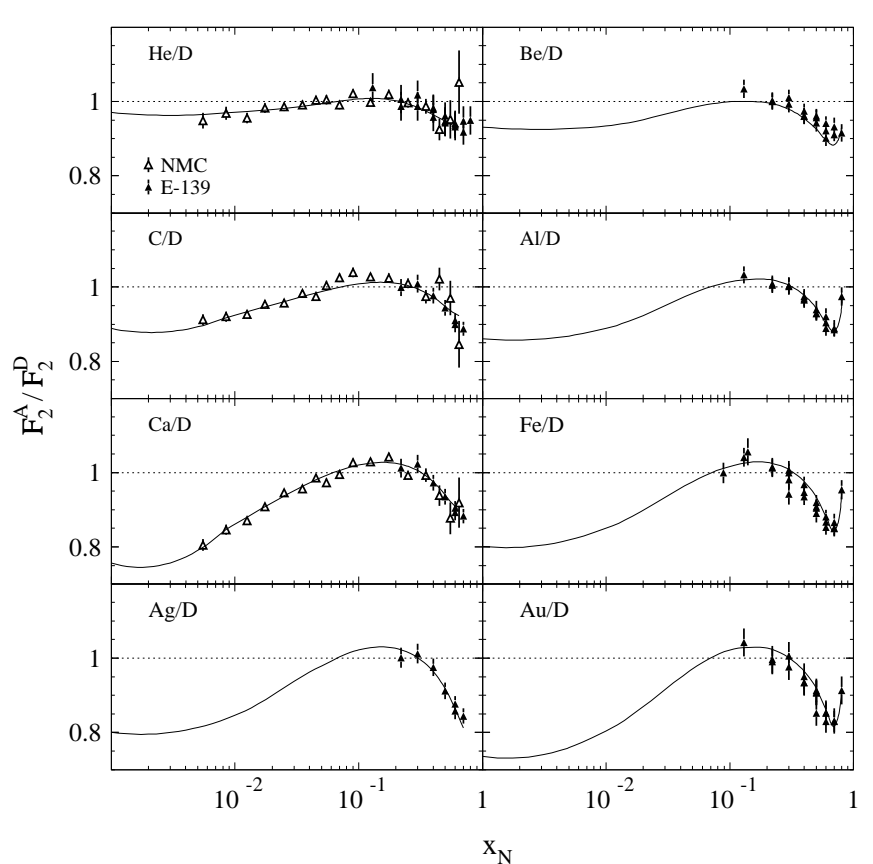

FIG. 1: $F_{2}^{A} / F_{2}^{D}$ data. The lines interpolate the values obtained with the NLO $n P D F$ set at the respective $Q^{2}$, and extrapolate to low $x_{N}$ at the $Q^{2}$ leftmost point.

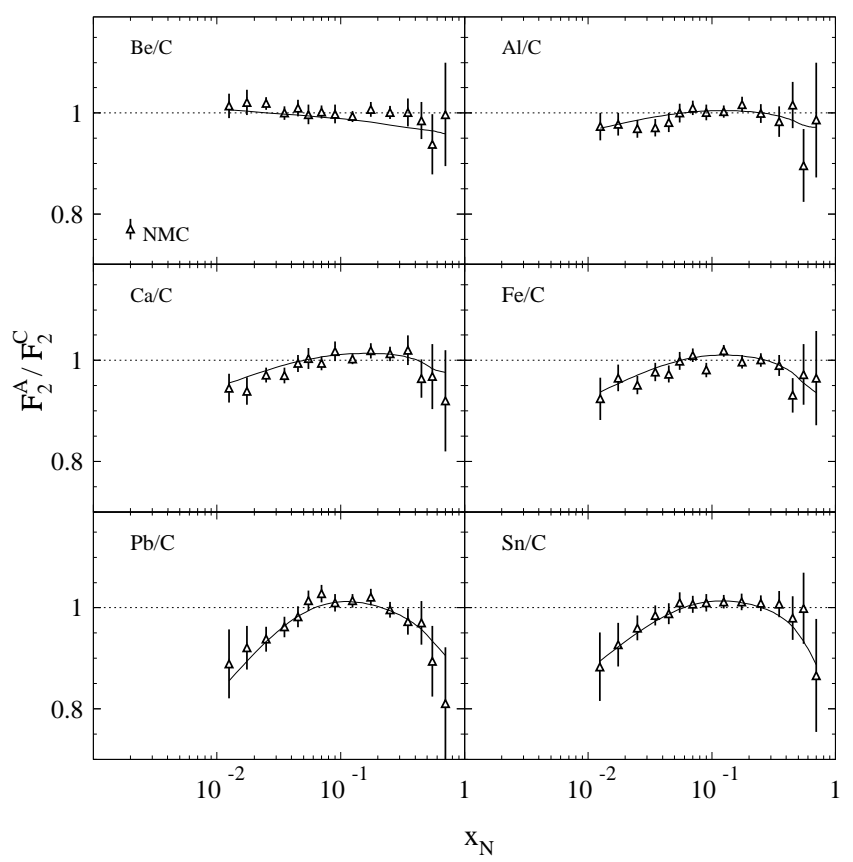

FIG. 2: The same as Fig. 1 but for $F_{2}^{A} / F_{2}^{C}$ data

enters at the lowest order, like in hadronic colliders, is needed to obtain a much better constraint.

The regular $A$ dependence of the parameters, as observed in Figure 5, helps to interpolate through regions where the data is scarce and also lead to reasonable extrapolations where there is not available.

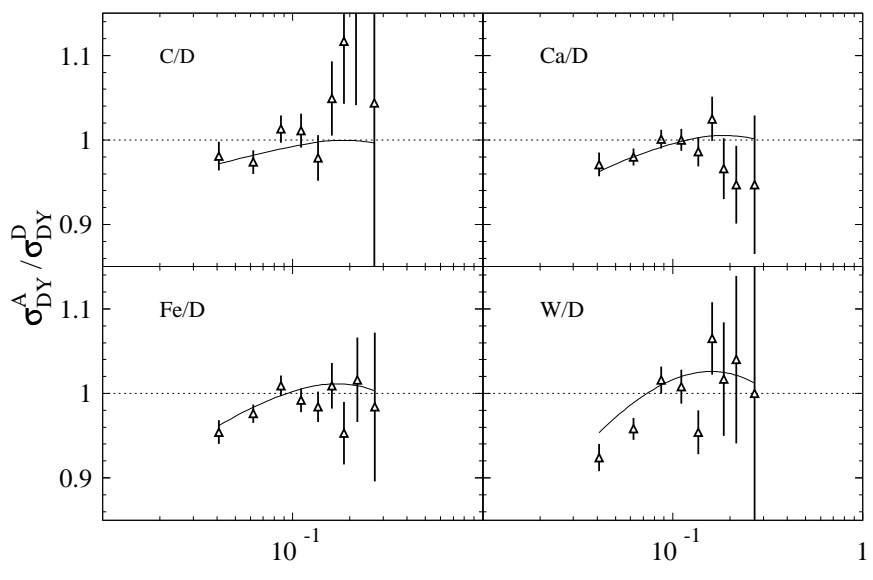

FIG. 3: Data on nuclear Drell $\stackrel{\mathrm{X}_{\mathrm{T}}}{\text { Yan }}$ cross sections rates to deuterium and those computed with NLO nPDF.

Noticeably, while some parameters show a clear dependence on the size of the nucleus, such as the shifts in the momentum fractions $\epsilon$ and $\epsilon^{\prime}$ which drive nuclear effects at moderate and large $x_{N}$, those related to the shape of the nucleus effective densities at small $x_{N}$, such as $\alpha_{v}$, $\alpha_{s}$ and $\alpha_{g}=\alpha_{s}$ are not strongly dependent on $A$. The well known $A$ dependence of shadowing effects at small $x_{N}$ is driven by the normalization of these effective densities $a_{s}$, and $a_{g}$, and also by the large $x_{N}$ behavior of the densities fixed by the parameters $\beta_{v}$ and $\beta_{s}$, which control how much of the large $x_{N}$ component of the PDF enters the convolution.

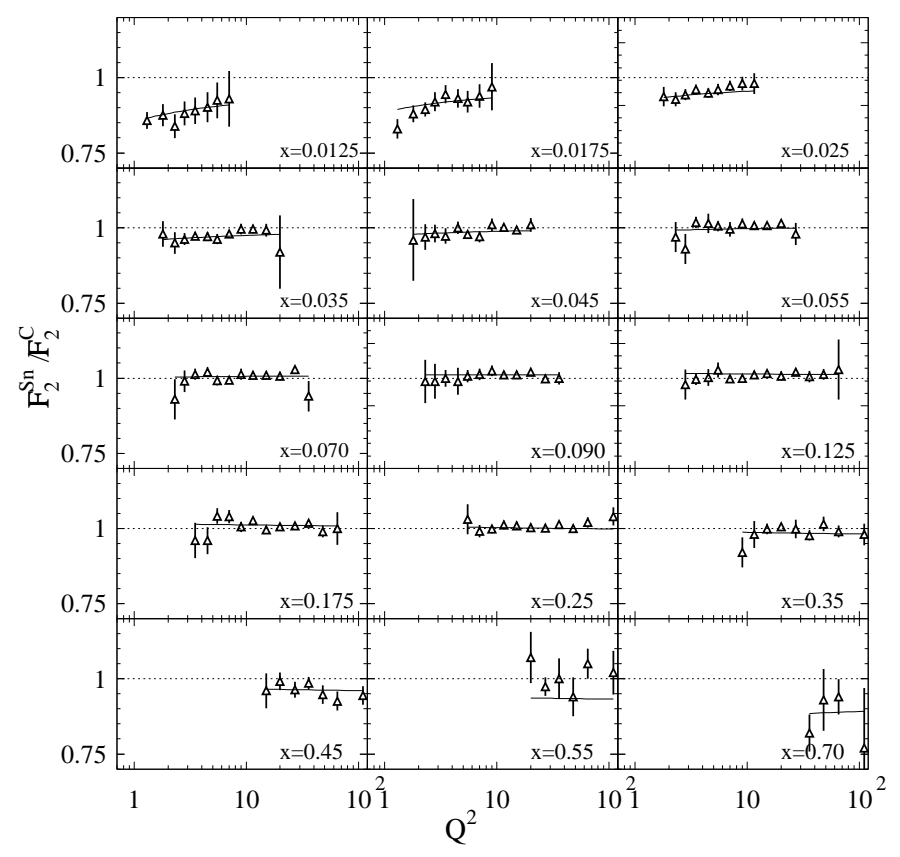

FIG. 4: Scale dependence of $F_{2}^{S n} / F_{2}^{C}$ data and the outcome of $N L O n P D F$. 


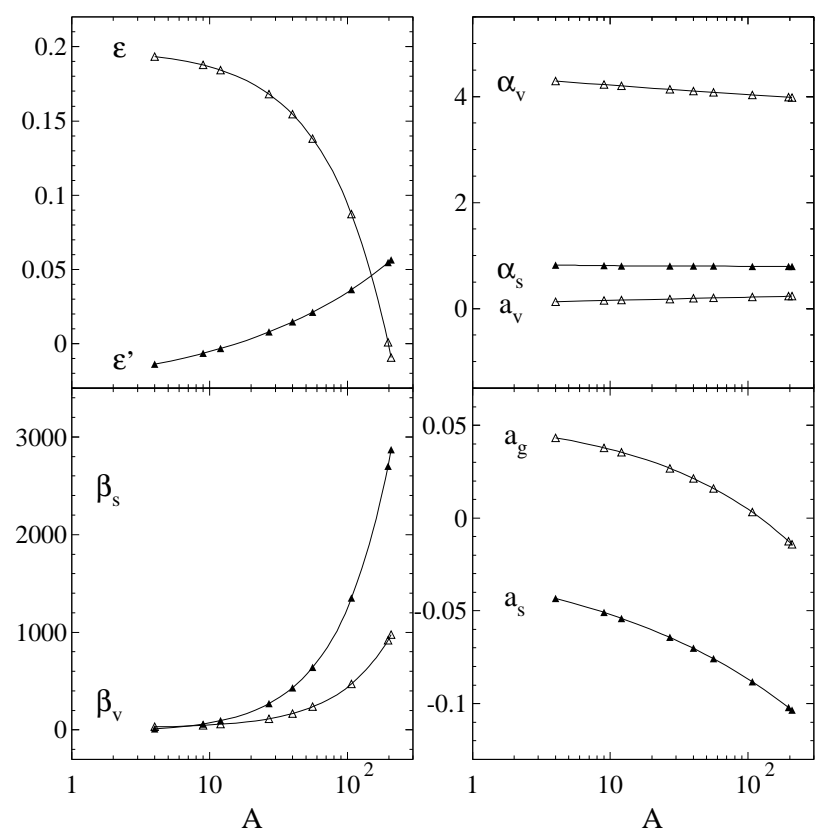

FIG. 5: A-dependence of the parameters.

The resulting nPDF are shown in Figure 6, as ratios to free proton PDF, as defined in Eq.(2), for valence quarks, light sea and strange quarks, and gluons, at $Q^{2}=10 \mathrm{GeV}^{2}$. The numerical computation of these ratios once the $\mathrm{nPDF}$ have been written and extracted within the convolution approach, is straightforward and allows a comparison with standard analyses, and other parton distribution functions. The ratios are also provided (in a FORTRAN code) as grids in $x_{N}, Q^{2}$ and $A$

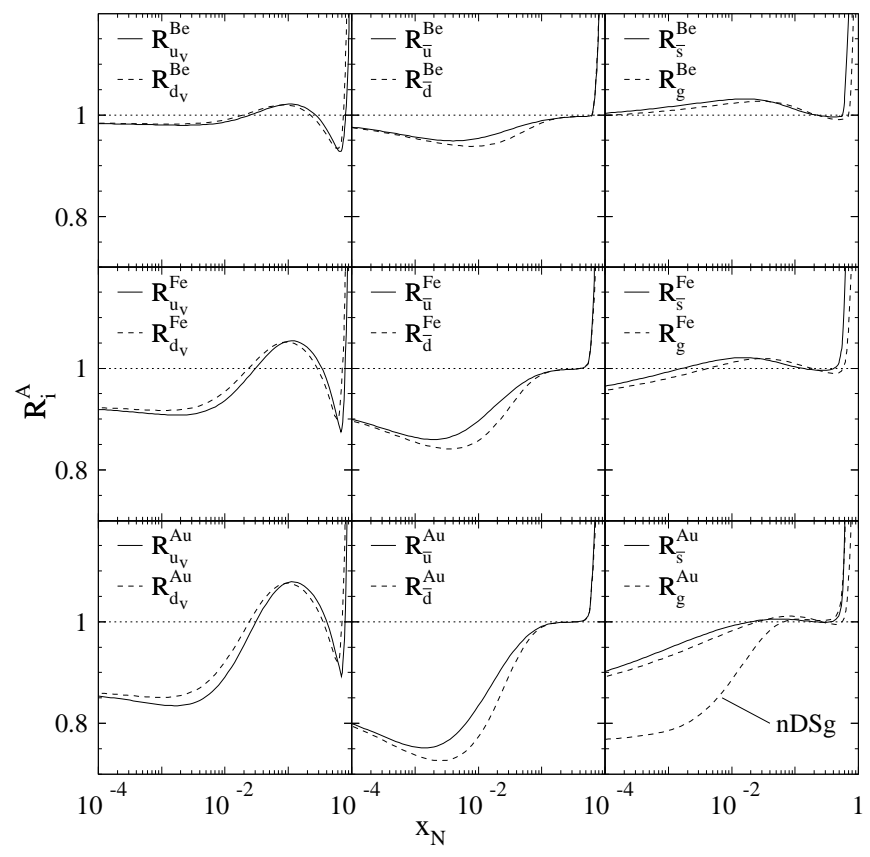

FIG. 6: The ratios for the densities are computed at $10 \mathrm{GeV}^{2}$

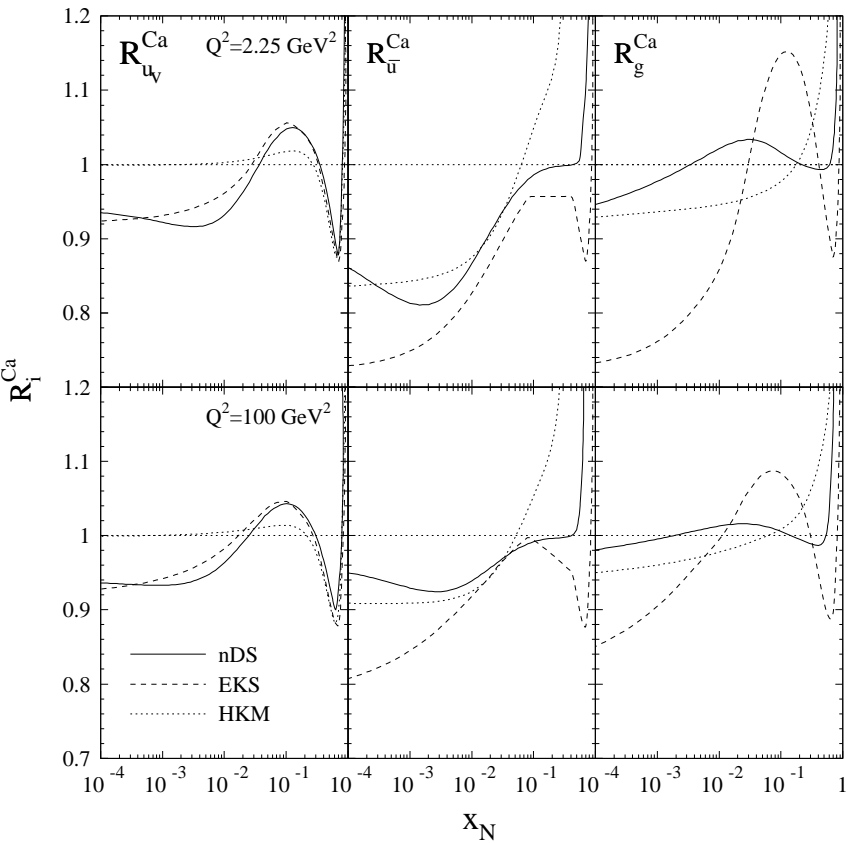

FIG. 7: Ratios coming from different $n P D F$ sets

for practical purposes and can be obtained upon request from the authors.

Similar results are found using nucleon parton densities other than GRV98. The similarity between modern parton densities guarantees that the nuclear ratios obtained with a given PDF set lead to reasonable nPDF when combined with another. Therefore, the parton distribution in a proton of a nucleus $A$ can be simply obtained by multiplying the nuclear ratios obtained in our analysis by any modern set of proton PDF. Of course, this is true provided the distributions come from analyses at the same order in QCD, as we will discuss in the next section.

It is worth mentioning that the agreement between nuclear parton distributions and data is remarkably better in the case of convolution based parameterizations than the ones found with multiplicative parameterizations in previous (LO) analyses. Those analyses yield $\chi^{2}$ values around 630 in the case of Ref. [6], and even larger values with the parameterization of Ref.[7], for the same data set used in the present analysis. A detailed comparison with the parameterization of Ref. [6] indicates that the $\chi^{2}$ of that set is basically due to a large contribution from the $Q^{2}$-dependence of $F_{2}^{S n} / F_{2}^{C}$ data. Therefore, one might expect significant differences between our LO gluon nuclear ratio and that of EKS98.

In Figure 7 we show a comparison between the nuclear ratios for LO $u_{\text {valence, }}, \bar{u}$ and $g$ proton (in calcium) distributions from nDS, EKS98 and HKM sets, at $Q^{2}=2.25$ $\mathrm{GeV}^{2}$ and $100 \mathrm{GeV}^{2}$.

The main differences with previous nuclear parameterizations are found in gluon densities, and to less extent in sea quarks, when comparing with ESK98, and in valence 
and sea quarks in the case of HKM. Our gluon densities show comparatively less small $x_{N}$ shadowing for heavy nuclei than ESK98, and very little antishadowing at intermediate $x_{N}$, which is considerable in their distributions. For nuclei lighter as $\mathrm{C}$ our gluons show only a tiny antishadowing effect.

In order to study the sensitivity of different observables on the amount of shadowing in the nuclear gluon distribution, we have performed an alternative extraction of $\mathrm{nPDF}$ from the same data set but constraining the gluon density in heavy nuclei to show a stronger shadowing effect at small $x_{N}$. We provide the result in set called nDSg, constrained to satisfy $R_{g}^{A u}=0.75$ at $x_{N}=0.001$ and $Q^{2}=5$. The $\chi^{2}$ value of this analysis is around 550 , considerably larger than the unconstrained fit, and should be considered only as a mean to study variations on, mainly, the gluon nuclear distribution. An example for the gluon nuclear ratio in $A u$ is shown in the last plot in Figure 6.

Compared with HKM, our valence distributions show low $x_{N}$ shadowing, whereas in HKM there is none. In our parameterization the ratios for sea and gluon densities approach unity as $x_{N}$ grows, while with HKM distributions these ratios show a strong rise. The difference in the fits may be understood due to the fact that both the low $x_{N}$ region in valence densities, and the large $x_{N}$ behavior of the sea distributions have little impact in DIS observables, and is only picked up by Drell-Yan yields, not included in HKM analysis.

\section{NLO}

Although there are no significant differences between the total $\chi^{2}$ values obtained in LO and NLO fits, the corresponding $\mathrm{nPDF}$, and also the ratios to ordinary $\mathrm{PDF}$, $R_{i}\left(x_{N}, Q^{2}, A\right)$, indeed differ, specially at low $Q^{2}$. In some cases, the differences are as large as the nuclear effects themselves. In Figure 8 the ratios between the NLO and LO extractions of $R\left(x_{N}, Q^{2}, A\right)$

$$
K_{i}^{A}\left(x_{N}, A, Q^{2}\right) \equiv \frac{R_{i}^{N L O}\left(x_{N}, Q^{2}, A\right)}{R_{i}^{L O}\left(x_{N}, Q^{2}, A\right)}
$$

are shown as a function of $x_{N}$ for various $Q^{2}$ and different nuclei.

The main differences between $R_{i}^{N L O}\left(x_{N}, Q^{2}, A\right)$ and $R_{i}^{L O}\left(x_{N}, Q^{2}, A\right)$ are found in sea quark and gluon densities, most noticeably at low $x_{N}$ and in heavy nuclei. These differences are correlated through the scale dependence with a similar behavior in the valence ratios at small $x_{N}$. As one would expect, the differences are more significant at small $Q^{2}$ and fades away as $Q^{2}$ increases.

The fact that LO analyses lead to fits comparable in accuracy to NLO analyses is due to the relatively moderated range in $Q^{2}$ spanned by the data, and the absence in the data set of nuclear observables strongly dependent on the gluon distributions. This, of course neither imply that the differences in $R\left(x_{N}, Q^{2}, A\right)$ as obtained at

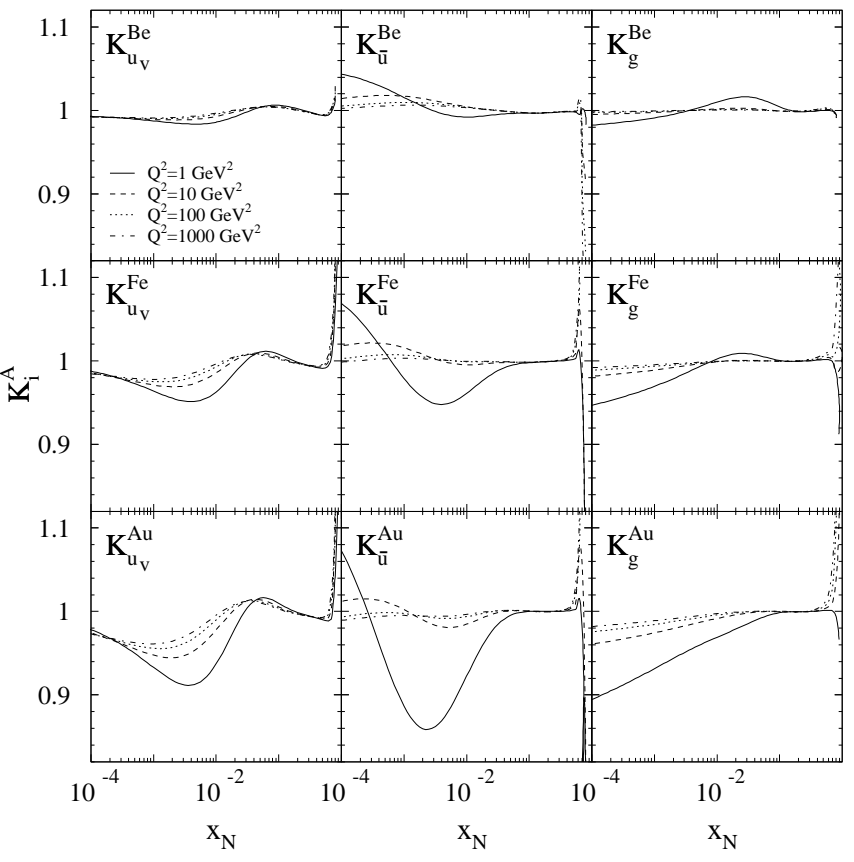

FIG. 8: Ratios between NLO and LO extractions of nuclear effects for different nuclei.

$\mathrm{LO}$ and at NLO accuracy would be negligible, in fact beyond LO the ratios are factorization scheme dependent, nor that there would not be significant NLO corrections in other observables. This is particularly true for those that rely on the gluon or sea quark densities and stress the importance of having NLO extractions of nPDF.

As an example of an observable which is sensitive to NLO corrections, in Figure 9 we show the LO and NLO leading twist cross sections for the production of neutral pions in $\mathrm{d}-\mathrm{Au}$ collisions as a function of the transverse momentum $p_{T}$ of the final state particle. The plots correspond to nucleon center of mass energies $\sqrt{s_{N N}}$ of 200 $\mathrm{GeV}$ and a range in pseudo-rapidity of $|\eta|<0.18$, computed using the code in [18] adapted for nuclear beams. Pion fragmentation functions were taken from Ref. 19]. The LO and NLO cross section are computed at two different values for the factorization and renormalization scales $\mu_{R}=\mu_{F}=p_{T}$ and $\mu_{R}=\mu_{F}=2 p_{T}$. The differences between both predictions give an estimate of the theoretical uncertainty in the fixed order calculation. As can be observed, there is a considerable reduction in the scale dependence of the cross section when the NLO corrections are taken into account. This feature is found in almost any infrared-safe observable in hadronic collisions, indicating that $\mathrm{LO}$ calculations can only provide a qualitative description. The inset in Figure 9 shows the $K_{N L O}$ factor, defined as

$$
K_{N L O}=\frac{\sigma_{d A u}^{N L O}}{\sigma_{d A u}^{L O}}
$$

i.e. the ratio between the NLO and LO cross section, computed at a given factorization and renormalization 


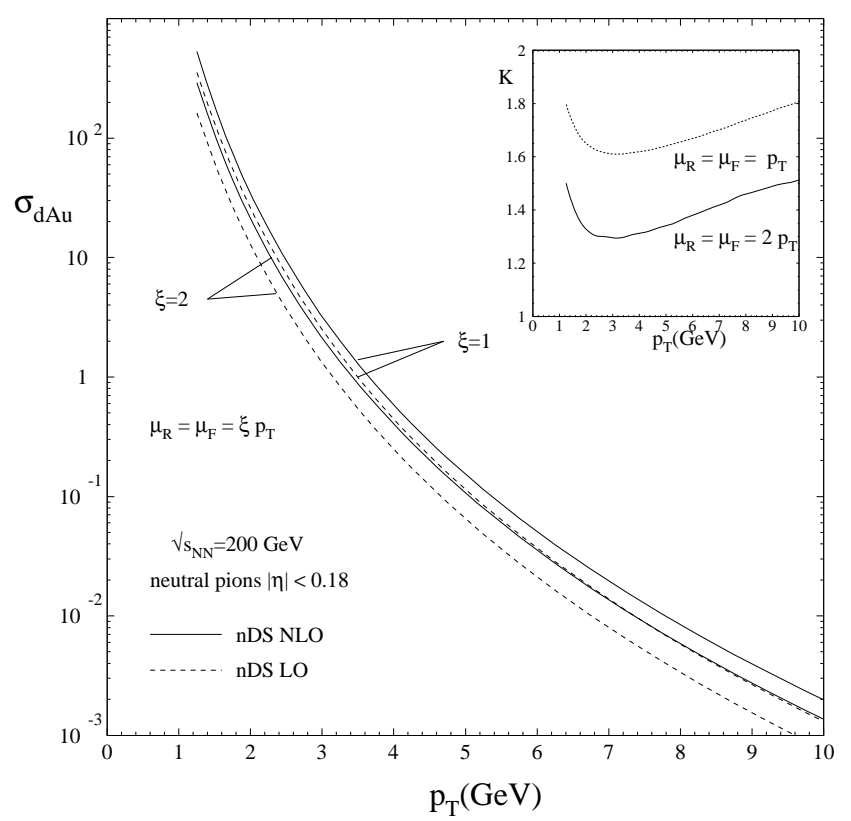

FIG. 9: LO and NLO neutral pion production cross sections for $d$-Au collisions.

scales, and using the corresponding $\mathrm{nPDF}$ and fragmentation functions. Notice that the $K_{N L O}$ factor is not a physical quantity, actually it turns out to be strongly scale dependent, but provides an estimate of the size of the NLO corrections. In this case, it shows that the oneloop QCD corrections for pion production at RHIC are of the order of $50 \%$ or even larger, while the strong increase in the correction when the transverse momentum $p_{T}$ is smaller than $\sim 2 \mathrm{GeV}$ indicates we approach the limit of the region of validity of $\mathrm{pQCD}$ calculations.

Finally, in Figure 10 we show the estimates for the d-Au cross sections to neutral pions but normalized to proton-proton cross sections, against the data reported by [20], and not included in the present fit. With the exception of the lowest $p_{T}$ data, which is in the borderline of the perturbative domain, the estimate agrees with the data well within the experimental uncertainties. Notice that even though the LO and NLO cross sections differ substantially, the discrepancies almost cancel in the ratios, to proton, highlighting the perturbative stability and the consistency between the LO and the NLO extractions of nPDF. To quantify the effect of nuclear shadowing in the gluon distribution on hadron production at RHIC we have computed the same observable using the alternative $\mathrm{NLO}$ set of $\mathrm{nPDF}$ (set $\mathrm{nDSg}$ ) with a larger shadowing in the gluon distribution at small $x_{N}$. The Figure shows a reduction in the d-Au cross section at small $p_{T}$ for the set with larger gluon shadowing, however it seems unlikely to obtain a much smaller value for the ratio $R_{d A U}$ at $p_{T} \sim 2 \mathrm{GeV}$ with realistic nPDF. Notice that the cross sections receives contributions typi-

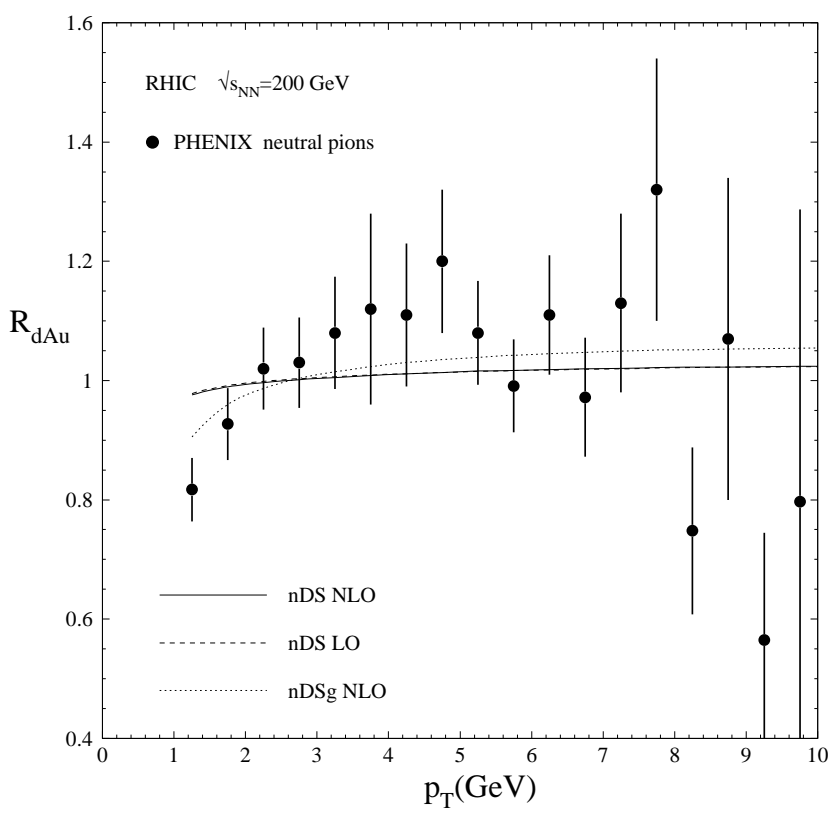

FIG. 10: Neutral pion production nuclear rates.

cally from $x_{N}>0.01$. Similar conclusions, with a slightly bigger reduction at small $p_{T}$, are reached if the same observable is computed for neutral pion production in the forward region $(\eta \sim 3)$. Any experimental finding on a stronger reduction of $R_{d A U}$ might certainly be considered as an evidence of a new phenomena, at least beyond pQCD.

\section{CONCLUSIONS}

We have performed for the first time a full NLO QCD global analysis of nuclear DIS and Drell Yan data using a convolution approach to parameterize nPDF. We have found that this strategy not only leads to much more accurate $\mathrm{nPDF}$ but simplifies considerably the numerical computation of QCD corrections at NLO. Although both LO and NLO nPDF reproduce the available data with comparable precision, they show non negligible differences which have to be taken into account when computing other observables.

\section{ACKNOWLEDGEMENTS}

We warmly acknowledge W. Vogelsang for interesting discussions and suggestions.

\section{APPENDIX}


TABLE II: Parameters of the NLO and LO nPDF

\begin{tabular}{|c|c|c|c|c|c|c|}
\hline \multirow[t]{2}{*}{ Parameter } & \multicolumn{3}{|c|}{$\mathrm{NLO}$} & \multicolumn{3}{|c|}{$\mathrm{LO}$} \\
\hline & $\gamma$ & $\lambda$ & $\delta$ & $\gamma$ & $\lambda$ & $\delta$ \\
\hline$\overline{\epsilon_{v}}$ & 0.1984 & -0.0013 & 0.0814 & 0.2030 & -0.0014 & 0.9510 \\
\hline$\epsilon_{v}^{\prime}$ & 0.0346 & -0.0124 & 0.9421 & 0.0351 & -0.0133 & 0.3657 \\
\hline$a_{v}$ & 0.7546 & -0.6687 & -0.0473 & 0.7251 & -0.6647 & -0.0583 \\
\hline$\alpha_{v}$ & 2.1412 & 2.2690 & -0.0390 & 2.1786 & 2.5720 & -0.0439 \\
\hline$\beta_{v}$ & -0.0474 & 0.3730 & 1.1301 & 19.925 & 2.2760 & 1.1463 \\
\hline$a_{s}$ & -0.0135 & -0.0202 & 0.2797 & -0.0179 & -0.0189 & 0.2664 \\
\hline$\alpha_{s}$ & 0.7980 & 0.0814 & -0.8647 & 1.0616 & 0.0572 & -0.6277 \\
\hline$\beta_{s}$ & -24.325 & 7.3191 & 1.1204 & -24.107 & 7.3526 & 0.4284 \\
\hline$a_{g}$ & 0.0565 & -0.0073 & 0.4244 & 0.0629 & -0.0076 & 0.4285 \\
\hline$\overline{\chi^{2} / d . o . f .}$ & & $299.91 / 393$ & & & $316.35 / 393$ & \\
\hline
\end{tabular}

[1] J. J. Aubert et al., Phys. Lett. B123, 275 (1983).

[2] M. Arneodo, Phys. Rep. 240301 (1994).

[3] S. S. Adler et al., Phys. Rev. Lett. 91, 072301 (2003); J. Adams et al., nucl-ex/030515.

[4] A. Accardi, et al. hep-ph/0308248

[5] E. H. Paschos, J. Y. Yu, Phys. Rev. D 65033002 (2002).

[6] K. J. Eskola, V. J. Kolhinen, P. V. Ruuskanen, Nucl. Phys. B535, 351 (1998); K. J. Eskola, V. J. Kolhinen, C. A. Salgado, Eur. Phys. J. C9, 61 (1999). B535, 351 (1998)

[7] M. Hirai, S. Kumano, M. Miyama, Phys. Rev. D 64 034003 (2001)

[8] C. A. García Canal et al., Phys. Rev. Lett.53 1430. (1984)

[9] D. de Florian et al., Z. Phys.A350 55 (1994).

[10] E. G. Floratos, D. A. Ross, C. T. Sachrajda, Nucl. Phys. B152 493 (1977); A. Gonzalez Arroyo, C. Lopez, F. J. Yndurain, Nucl. Phys. B153 161 (1979); G. Curci, W.
Furmanski, R. Petronzio, Nucl. Phys. B175 257 (1980).

[11] M. Glück, E. Reya, A. Vogt, Eur. Phys. J. C5, 461 (1998).

[12] F. Close, J. Qiu, R. G. Roberts, Phys. Rev. D 402820 (1989).

[13] P. Amaudruz et. al., Nucl. Phys. B441, 3 (1995); M. Arneodo et al., B441, 12 (1995).

[14] J. Gomez et al., Phys. Rev. D 49, 4348 (1994).

[15] M. Arneodo et al., Nucl. Phys. B481, 3 (1996).

[16] M. Arneodo et al., Nucl. Phys. B481, 23 (1996).

[17] D. M. Alde et al., Phys. Rev. Lett. 64, 2479 (1990).

[18] D. de Florian, Phys. Rev. D 67, 054004 (2003).

[19] S. Kretzer, Phys. Rev. D 62054001 (2000).

[20] S. S. Adler et al., nucl-ex/0306021

[21] Notice that this is not the case for the ratios $R$ defined in $x$-space as in Eq. (2) 